\title{
Axially Extended-Volume C-Arm CT Using a Reverse Helical Trajectory in the Interventional Room
}

\author{
Zhicong Yu*, Andreas Maier, Günter Lauritsch, Florian Vogt, Manfred Schönborn, Christoph Köhler, \\ Joachim Hornegger, and Frédéric Noo
}

\begin{abstract}
C-arm computed tomography (CT) is an innovative technique that enables a $\mathrm{C}$-arm system to generate 3 -D images from a set of 2-D X-ray projections. This technique can reduce treatment-related complications and may improve interventional efficacy and safety. However, state-of-the-art $\mathrm{C}$-arm systems rely on a circular short scan for data acquisition, which limits coverage in the axial direction. This limitation was reported as a problem in hepatic vascular interventions. To solve this problem, as well as to further extend the value of C-arm CT, axially extended-volume $\mathrm{C}$-arm CT is needed. For example, such an extension would enable imaging the full aorta, the peripheral arteries or the spine in the interventional room, which is currently not feasible. In this paper, we demonstrate that performing long object imaging using a reverse helix is feasible in the interventional room. This demonstration involved developing a novel calibration method, assessing geometric repeatability, implementing a reconstruction method that applies to real reverse helical data, and quantitatively evaluating image quality. Our results show that: 1) the reverse helical trajectory can be implemented and reliably repeated on a multiaxis $\mathrm{C}$-arm system; and 2) a long volume can be reconstructed with satisfactory image quality using reverse helical data.
\end{abstract}

Index Terms-Axially extended field-of-view, C-arm computed tomography (CT), cone-beam image reconstruction, reverse helix.

\section{INTRODUCTION}

C -ARM COMPUTED tomography (CT) is an X-ray cone-beam (CB) imaging technique that enables a $\mathrm{C}$-arm system to generate isotropic 3-D images in the interventional room. Recent clinical reports [1]-[6] demonstrate that this technique is valuable for various tasks in the interventional room.

Manuscript received July 01, 2014; revised August 15, 2014; accepted August 15, 2014. Date of publication August 27, 2014; date of current version December 24, 2014. This work was supported in part by a grant of Siemens AG, Healthcare and by the U.S. National Institutes of Health (NIH) under Grant R21 EB009168 and R01 EB007236. Its contents are solely the responsibility of the authors and do not necessarily represent the official views of the NIH. The concepts presented in this paper are based on research and are not commercially available. Asterisk indicates corresponding author.

*Z. Yu was with the Department of Radiology, University of Utah, Salt Lake City, UT 84112 USA. He is now with the Department of Radiology, Mayo Clinic, Rochester, MN 55901 USA

G. Lauritsch, F. Vogt, M. Schönborn, and C. Köhler are with Siemens AG, Healthcare, 91301 Forchheim, Germany.

A. Maier and J. Hornegger are with the Pattern Recognition Lab, University of Erlangen-Nuremberg, 91054 Erlangen, Germany.

F. Noo is with the Department of Radiology, University of Utah, Salt Lake City, UT 84112 USA.

Color versions of one or more of the figures in this paper are available online at http://ieeexplore.ieee.org.

Digital Object Identifier 10.1109/TMI.2014.2350986
For example, C-arm CT is used for localizing and assessing chemoembolization as well as for placing stent grafts for aortic aneurysms [3], [4], [1].

Currently, C-arm CT uses a circular short scan for data acquisition. This setup results in undesirable CB artifacts [7], [8] and only provides short coverage in the direction of the patient table, which we call the axial direction. The short coverage is limiting for hepatic vascular interventions [6], as well as for the treatment of aortic diseases and structural damage in the vertebral body. This paper focusses on extending the length of the field-of-view (FOV) in C-arm CT while using a trajectory that allows exact and stable reconstruction.

Extended-volume $\mathrm{C}$-arm CT is challenging because a $\mathrm{C}$-arm system can only rotate in a given direction over a limited angular range, which is usually much less than $360^{\circ}$. In particular, the helical trajectory that is commonly used in CT scanners is difficult to implement on a $\mathrm{C}$-arm system. The helix could be created as shown in [9]. However, this method requires the C-arm to rotate in each direction at least $360^{\circ}$, which is not always possible; furthermore, it requires a long pause in X-ray exposure that increases scan time and yields discontinuities in the data acquisition that can be inconvenient when imaging with contrast agent. The reverse helix that was proposed for extended-volume imaging in image-guided radiation therapy [10] may be more attractive for extended-volume C-arm CT. Here, we demonstrate that the reverse helix can be robustly implemented on a $\mathrm{C}$-arm system. Because the reverse helix satisfies Tuy's condition [11], [12], this demonstration implies that exact and stable reconstruction is also possible. However, this aspect is not our focus as it was already nicely illustrated in [7] with real data from an On-Board Imaging device in radiation therapy. Note that several reconstruction algorithms for the reverse helix have been reported [10], [13]-[15], and preliminary results related to our work were presented in [16].

A number of considerations were taken into account in the design of our demonstration. First, we chose to implement the reverse helix on a multiaxis $\mathrm{C}$-arm system so that the rotationplus-translation motion can be solely realized by the $\mathrm{C}$-arm. The patient bed was kept stationary for two reasons: 1) moving the patient may not be practical in the middle of a surgical intervention; 2) the floating patient table that is often used in the interventional room is not able to provide accurate translation motion. Second, an accurate geometrical calibration had to be developed to enable reconstruction, and also to identify how close the realized motion is to the prescribed reverse helix. Many 
calibration methods for a single circular trajectory are available in the literature (see, e.g., [17]-[24]), however, none of these methods was readily applicable to reverse helical scanning. Third, attention had to be paid to motion repeatability, because it is difficult to perform a single scan that simultaneously provides information for trajectory calibration and tomographic data of the patient. C-arm CT usually performs trajectory calibration once and uses the calibrated trajectory in subsequent tomographic scans. Fourth, there is the issue of data corrections that are typically applied to handle physical limitations in the data acquisition, such as beam-hardening, truncation, scatter and overexposure. Finding suitable corrections requires extensive investigations. Such corrections have been developed for circular CB imaging [25]. Here, we decided to restrict our study to examining how well these developed corrections apply to reverse helical data. Finally, there was the question of how to perform reconstruction, particularly under the condition that the data geometry would most likely slightly deviate from an ideal reverse helix. Exact algorithms such as those presented in [14], [13] can be modified to account for such deviations, but such effort was deemed too demanding at this stage of our investigation. Instead, we decided to adapt the approximate Fusion-RFDK method [15] for which geometrical deviations are much easier to handle. As mentioned above, demonstrating the advantage of data completeness associated with the reverse helix was not our focus.

The manuscript is organized as follows. First, we discuss data acquisition in Section II. This discussion includes a brief introduction to the multiaxis system being used, a description of the reverse helix being implemented, a description of phantoms chosen for our demonstration, and a presentation of reverse helical data for one of these phantoms, demonstrating the long axial coverage achieved by the reverse helix. Section III presents the calibration method that was developed to assess the geometry of the implemented reverse helix, and also presents results demonstrating robustness in motion repeatability. Section IV presents the reconstruction algorithm and discusses practical issues associated with its implementation. Section V presents reconstructions from the real reverse helical data together with a quantitative comparison against results from a short circular CB scan. The primary purpose of the comparison is to show that long FOV imaging with the reverse helix is achieved with no loss in image quality compared to what is routinely used in the clinic. Finally, Section VI summarizes our results and discusses future directions.

\section{EXPERIMENTAL SETUP}

In this section, we first describe the multiaxis C-arm system that was used for our experiments, then provide implementation details for a five-turn reverse helix. Next, we describe the testing phantoms, scanning parameters as well as data correction methods.

\section{A. C-Arm System}

The multiaxis C-arm system (Artis zeego, Siemens AG, Healthcare, Forchheim, Germany [26, Fig. 1] is a floor-mounted robotic arm at the end of which is attached a conventional $\mathrm{C}$-arm. This $\mathrm{C}$-arm is equipped with a flat panel detector of
TABLE I

PARAmeters USED FOR THE REVERSE HeliCAL TRAJECTORY WiTH THE MUlTiAXIS C-ARM SYSTEM

\begin{tabular}{ll}
\hline \hline Parameter & Value \\
\hline Source-to-detector distance & $1200 \mathrm{~mm}$ \\
Detector A/D converter & $16 \mathrm{bits}$ \\
Detector size & $300 \mathrm{~mm} \times 400 \mathrm{~mm}$ \\
Detector position mode & landscape \\
Detector pixel size & $154 \mu \mathrm{m} \times 154 \mu \mathrm{m}$ \\
Detector binning mode & $2 \times 2$ \\
Source-to-rotational-axis distance & $785 \mathrm{~mm}$ \\
Axial height of the helical turns & $60 \mathrm{~mm}$ \\
Angular length per turn & $240^{\circ}$ \\
Angular step size & $0.35^{\circ}$ \\
Number of samples per turn & 681 \\
\hline \hline
\end{tabular}

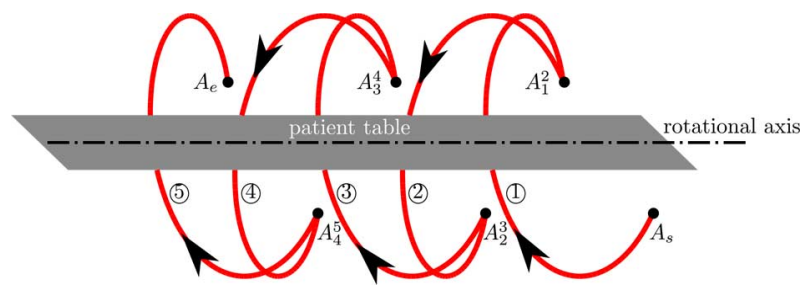

Fig. 1. Five-turn reverse helix. This reverse helix travels from $A_{s}$ to $A_{e}$ and alternates the direction of rotation at kink points $A_{1}^{2}, A_{2}^{3}, A_{3}^{4}$, and $A_{4}^{5}$. Circled numbers indicate the order of all helical turns. Rotational axis is viewed as the long axis of the patient table.

size $300 \mathrm{~mm} \times 400 \mathrm{~mm}$ and an X-ray source that is at a fixed distance of $1200 \mathrm{~mm}$ from the detector. The detector can be positioned in two modes: the landscape mode and the portrait mode. In our experiment, we adopted the landscape mode to maximize the radius of the FOV in the transverse direction, which was approximately $130 \mathrm{~mm}$.

The flat panel detector consists of $1920 \times 2480$ pixels of size $154 \mu \mathrm{m} \times 154 \mu \mathrm{m}$ with a 16 -bit A/D converter. Three modes are available for binning: $1 \times 1,2 \times 2$, and $4 \times 4$. In our experiment, the $2 \times 2$ binning mode was selected, so that the effective pixel size was $308 \mu \mathrm{m} \times 308 \mu \mathrm{m}$. These data-acquisition-related parameters are listed in Table I.

\section{B. Reverse Helix Configuration}

The reverse helix consists of multiple consecutive helical turns. These helical turns share the same translation direction, rotational axis, rotational radius, as well as angular length. However, any two consecutive helical turns of the reverse helix are composed of one left-handed helical turn and one right-handed helical turn, and these two turns are connected at a unique point called the kink point. For a mathematical definition of the reverse helix, we refer to [10], [13], and [15].

We implemented a five-turn reverse helix, which is depicted in Fig. 1. This reverse helix starts at $A_{s}$, ends at $A_{e}$, and includes four reversals in the rotational direction, at the kink points $A_{1}^{2}$, $A_{2}^{3}, A_{3}^{4}$, and $A_{4}^{5}$. The circled numbers indicate the order of the helical turns: the first, third, and fifth turns are left handed, whereas the second and fourth turns are right handed.

The five-turn reverse helix was implemented by rotating and translating the $\mathrm{C}$-arm of the multiaxis system around the patient table, which was kept stationary. The rotational radius and the angular length of each helical turn was set to be $785 \mathrm{~mm}$ and $240^{\circ}$, respectively. Along each helical turn, 681 X-ray source 
TABLE II

Dose-Area-Product Measurement for the Five-Turn REVERSE HELIX $\left(\mathrm{MGY} \cdot \mathrm{CM}^{2}\right)$

\begin{tabular}{l|lllll|l}
\hline \hline & 1 st & 2nd & 3rd & 4th & 5th & total \\
\hline Torso & 60.27 & 60.60 & 60.59 & 60.61 & 60.64 & 302.70 \\
\hline CATSCB & 314.60 & 315.54 & 315.71 & 315.65 & 315.41 & 1576.91 \\
\hline \hline
\end{tabular}

positions were uniformly sampled using an angular step size of $0.35^{\circ}$. Let the axial height of any helical turn on the reverse helix be the distance between the orthogonal projection of its endpoints onto the rotational axis. The axial height of all helical turns was set to be $60 \mathrm{~mm}$. The above mentioned parameters are listed in Table I.

It is important to note that $\mathrm{C}$-arm systems are not able to produce perfect source trajectories due to their open design. The exact X-ray source positions need to be estimated using a trajectory calibration process. The configuration parameters listed in Table I were only input values to the control system of the multiaxis C-arm system; systematic deviations and statistical fluctuations should be expected between the input and calibrated parameters.

\section{Phantoms and Data Generation}

We selected two phantoms for data acquisition. The first one is a torso phantom embedded with a SAWBONES spine (Sawbones Europe AB, Malmö, Sweden). The torso phantom was made of foam and was of size $400 \mathrm{~mm} \times 200 \mathrm{~mm} \times 550 \mathrm{~mm}$, whereas the SAWBONES spine was of length $360 \mathrm{~mm}$. The torso phantom was placed in the prone position and so that the entire SAWBONES spine was within the FOV. Projection data of the torso phantom were truncated in both the axial and transverse directions due to the limited size of the panel detector. However, data truncation in the transverse direction with this phantom is known to have little impact on image reconstruction, because the attenuation coefficient of the foam surrounding the SAWBONES spine is close to that of air (namely, about $-800 \mathrm{HU}$ at $90 \mathrm{kVp}$ ).

The second phantom is composed of the the CATPHAN phantom (The Phantom Laboratory, Greenwich, NY, USA) and the Siemens CB phantom (QRM, Möhrendorf, Germany) that were placed in-line along their axial direction to emulate a long object. We call this combination the CATSCB phantom. The CATSCB phantom was positioned along the patient table so as to fully fit within the FOV, hence the projection data for the CATSCB phantom were truncated in the axial direction but not in the transverse direction.

The torso phantom was scanned using $(90 \mathrm{kVp}, 43 \mathrm{~mA})$ with average pulsewidth $3.1 \mathrm{~ms}$, whereas the CATSCB phantom was scanned using $(91.2 \mathrm{kVp}, 122.8 \mathrm{~mA})$ with average pulsewidth $7.3 \mathrm{~ms}$. The dose-area-product measurement for each turn of the reverse helix is listed in Table II. In each case, no beam filtration was used, and an antiscatter grid was placed in front of the detector. The raw data of both phantoms were first transformed by a logarithm operation to estimate the line integrals of the X-ray attenuation coefficient. To account for classical physical limitations in the data acquisition process, three corrections based on the methods presented in [25] were subsequently applied to the

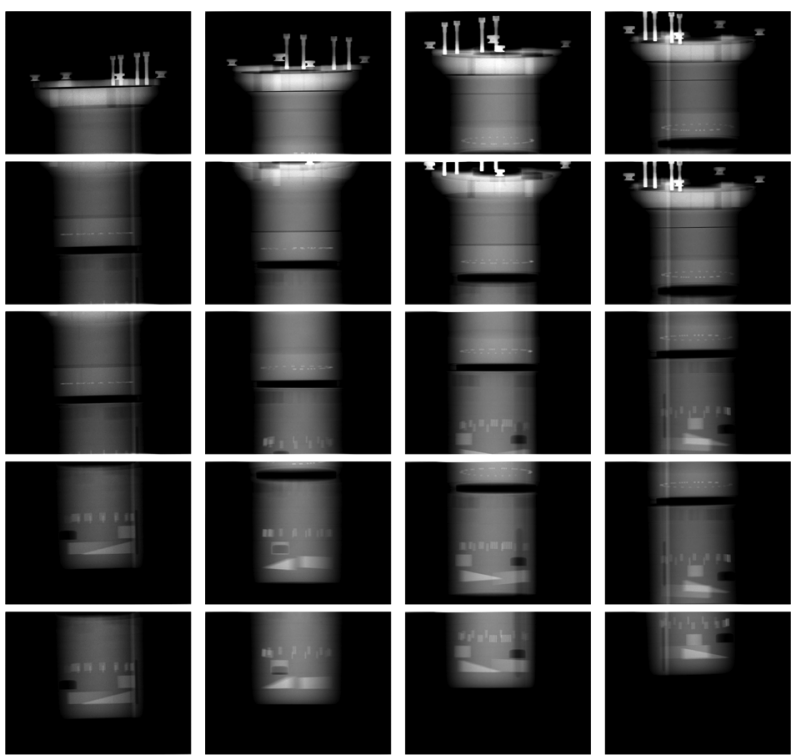

Fig. 2. Preprocessed projections of the CATSCB phantom. Three data corrections were applied: overexposure correction, scatter correction and beam hardening correction. Top to bottom: the first to the fifth helical turn. Each column presents projections at the same azimuthal angular position. The angular distances from the second, third, and fourth columns to the first column are $80^{\circ}$, $160^{\circ}$, and $230^{\circ}$, respectively.

line integrals: 1) overexposure correction; 2) scatter correction; and 3) beam hardening correction. Note that these corrections are optimized for a circular data acquisition.

Preprocessed projection data of the CATSCB phantom (Fig. 2) show that continuous scanning with great coverage along the axial direction was achieved by the reverse helical trajectory. Similar observations were made for the SAWBONES spine of the torso phantom.

\section{TRAJECTORY CALIBRATION}

Because of its open design, a C-arm system cannot produce a perfect source trajectory. The source and the detector positions produced by a C-arm system always need to be estimated by a calibration process. Several methods have been suggested to calibrate a circular trajectory for C-arm systems (see, e.g., [17]-[24]), but none of them is ready to use for the reverse helix. To geometrically calibrate a reverse helix, we decided to extend the method in [22], [23], which has been reliably used in clinical settings. Hereafter, we describe this extension, and show results obtained with it. Then, we discuss robustness in repeatability, which is crucial for practical utilization of the reverse helix, as joint scanning of the patient and the calibration phantom is not convenient and may not even be possible in clinical routine.

\section{A. CB Geometry and Coordinate Systems}

All vectors are denoted using an underlined lowercase letter. A vector may also be referred to by its components (or coordinates for a position vector). Thus, $\underline{x}$ could be identified as $(x, y)^{T}$ in 2-D, or as $(x, y, z)^{T}$ in 3-D. Fig. 3 illustrates our CB geometry. A flat panel detector is used. The current source position is denoted as $O_{\mathrm{s}}$, the orthogonal projection of $O_{\mathrm{s}}$ onto the detector as $O_{\mathrm{d}}$, and the source-to-detector distance $\left(\left|O_{\mathrm{s}} O_{\mathrm{d}}\right|\right)$ as 


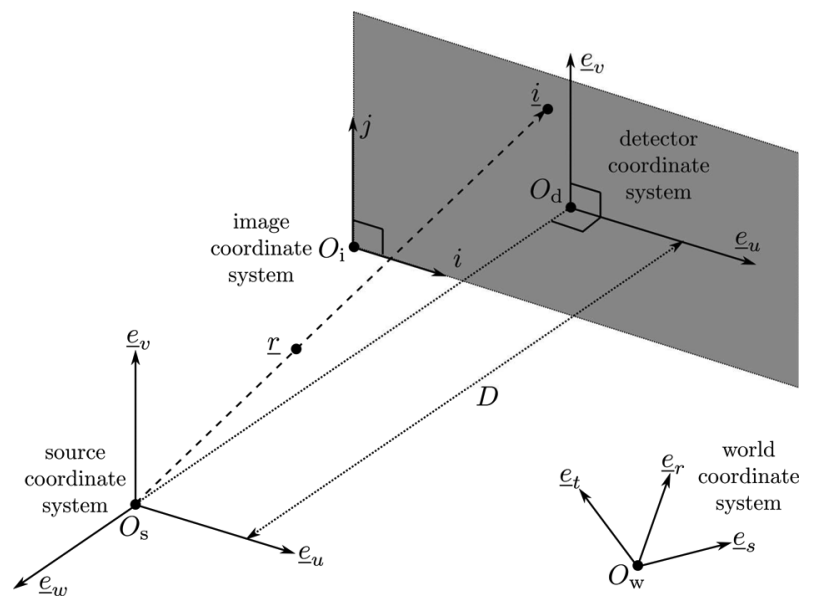

Fig. 3. Cone-beam geometry with four coordinate systems. Origins of the world coordinate system, the source coordinate system, the detector coordinate system and the image coordinate system are located at $O_{\mathrm{w}}, O_{\mathrm{s}}, O_{\mathrm{d}}$, and $O_{\mathrm{i}}$, respectively. $O_{\mathrm{s}}$ is the current source position and $O_{\mathrm{d}}$ is the orthogonal projection of $O_{\mathrm{s}}$ onto the detector. Distance between $O_{\mathrm{s}}$ and $O_{\mathrm{d}}$ is $D$. Here, $\underline{r}$ is a point in the world coordinate system and $\underline{i}$ is the CB projection of $\underline{r}$ in the image coordinate system as created from $O_{\mathrm{s}}$.

$D$. Also, the unit vector from $O_{\mathrm{d}}$ to $O_{\mathrm{s}}$ is called $\underline{e}_{w}$ and the detector plane is spanned by two orthonormal vectors, $\underline{e}_{u}$ and $\underline{e}_{v}$, such that $\underline{e}_{u} \times \underline{e}_{v}=\underline{e}_{w}$.

Geometrically, the data acquisition can be fully described with four coordinate systems. The first one, called the world coordinate system, is defined by a calibration phantom. It is of origin $O_{\mathrm{w}}$ and is spanned by three orthonormal vectors, $\underline{e}_{r}, \underline{e}_{s}$, and $\underline{e}_{t}$. Any point in this system can be identified by $(r, s, t)$ coordinates. The second one is the source coordinate system, the origin of which is at the current X-ray source position $O_{\mathrm{s}}$. It is spanned by $\underline{e}_{u}, \underline{e}_{v}$, and $\underline{e}_{w}$. The third one is the detector coordinate system. Its origin is at $O_{d}$ and it is spanned by $\underline{e}_{u}$ and $\underline{e}_{v}$. Any point in the detector plane can be identified by $(u, v)$ coordinates in this system. The fourth one is the image coordinate system, which is introduced to describe the detector pixel grid where the projection data are primarily presented. This system is spanned by $i$-and $j$-axes that are parallel to $\underline{e}_{u}$ and $\underline{e}_{v}$, respectively, with origin, $O_{\mathrm{i}}$, chosen as one corner of the detector. Each position vector $\underline{i}$ in the image coordinate system is expressed in pixel units.

\section{B. Calibration}

Consider a point $\underline{r}$ in the world coordinate system and its $\mathrm{CB}$ projection $\underline{i}$ in the image coordinate system. As discussed in [22], these two points, $\underline{r}$ and $\underline{i}$, can be related by a projection matrix $P$ that contains all needed $\mathrm{CB}$ geometrical information: source position, detector position and detector orientation. The calibration process aims at estimating the projection matrix $P$ for each source position.

To estimate the projection matrix $P$, enough pairs of $\underline{r}$ and $\underline{i}$ points have to be identified for each source position. For circular CB imaging, this goal can be achieved using the PDS-2 phantom [22], [27] that contains specially arranged spherical balls. Positions of these balls in the world coordinate system can be obtained from the manufacturer and positions of the projections of these balls in the image coordinate system can be

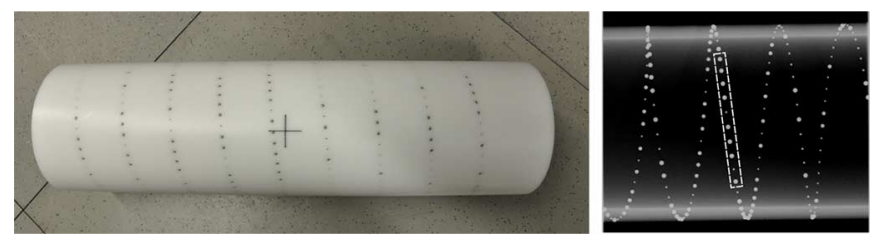

Fig. 4. Left: Siemens PDS-3 phantom with 10-bit encoding. Right: projection of the calibration phantom. Ten balls inside the rectangle identifies a unique number.

obtained using image segmentation techniques. The special arrangement of the balls involves an 8-bit encoding scheme that allows linking the projection of the balls to their positions in the calibration phantom. Unfortunately, the PDS-2 phantom is only $206 \mathrm{~mm}$ long and thus too short for long object imaging. To calibrate the reverse helix, a modified PDS-2 phantom that uses a 10 -bit encoding scheme was developed. This phantom is called the PDS-3 phantom.

The PDS-3 phantom is shown in Fig. 4 (left). It consists in a 450-mm-long cylindrical wall that contains 400 spherical balls. The balls are uniformly distributed on a helix of radius about $70 \mathrm{~mm}$. Half of the balls have a diameter of $3.2 \mathrm{~mm}$ and the other half have a diameter of $1.6 \mathrm{~mm}$. A ball of large radius represents a logic 1 , whereas a ball of small radius represents a logic 0 . The 400 balls are arranged on the helix such that any sequence of 10 consecutive balls corresponds to a unique number, which defines the position of each ball in the sequence. For example, in Fig. 4 (right), the 10 balls in the dashed box represents a unique binary sequence, 1111011101, which gives an index in a table that specifies where the balls of the sequence are located within the phantom.

The cylindrical wall of the phantom was manufactured with a state-of-the-art computer-numerical-control device (OKUMA LB400M-C1250, Abraham GmbH, Burgthann-Oberferrieden, Germany) that guaranteed an accuracy of about $5 \mu \mathrm{m}$ in positioning of the holes where the balls were inserted. The balls were made of stainless steel with a reported accuracy of $19 \mu \mathrm{m}$. The holes were of conical shape and of size slightly smaller than the ball size. The balls were inserted with pressurization so that they could hold in place by internal forces. The placement accuracy achieved with pressurization was of about $20 \mu \mathrm{m}$.

To make proficient use of the 10-bit-encoding feature, the PDS-3 phantom should be placed in the center of the FOV such that its centerline is as parallel as possible to the patient table. Note that the centerline of the PDS- 3 phantom is the $t$-axis. This configuration ensures detection of a good number of 10-bit sequences so that enough pairs of vectors $\underline{r}$ and $\underline{i}$ can be obtained (at least 11 pairs are needed but more pairs are useful to improve accuracy). Using the optimization method in [22], projection matrix $P$ can be reliably computed for any view for which enough pairs are obtained, and the CB geometrical information can be extracted from $P$ as explained in [28].

Based on the above calibration principles, we obtained the calibrated reverse helical trajectory in the $(r, s, t)$ world coordinate system; see Fig. 5. The calibrated reverse helical trajectory moves downwards along the $t$-axis; in the right of Fig. 5, the first helical turn appears on the top and the fifth helical turn appears on the bottom. The orthogonal projection (in the top 

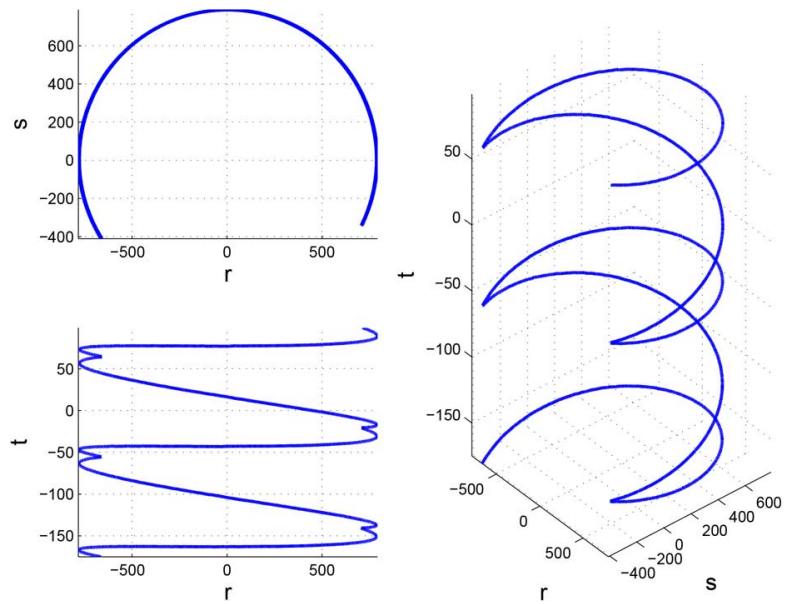

Fig. 5. Calibrated reverse helix. Top left: orthogonal projection onto the $(r, s)$-plane; bottom left: orthogonal projection onto the $(r, t)$-plane; right: 3 -D view. Trajectory moves downwards along the $t$-axis. Unit used for each axis: $\mathrm{mm}$.

left of Fig. 5) of the calibrated trajectory onto the $(r, s)$-plane yields five arcs that are very close to each other, indicating that the rotation axis used for the reverse helix is fairly close to the $t$-axis. However, these two axes are not aligned, as can be observed from the orthogonal projection of the trajectory onto the $(r, t)$-plane (Fig. 5, bottom left). If the two axes were aligned, the projection of the left handed turns would not have a slope close to zero. Since the calibration phantom was (roughly) aligned with the patient table, we can conclude that we succeeded in creating a reverse helix that would enable scanning the patient along the table.

\section{Trajectory Repeatability}

We now examine how well the reverse helical trajectory can be repeated. Consider two reverse helical trajectories that are calibrated with the same PDS-3 phantom placed at the same location with the same orientation. We analyze the similarity of these two calibrated trajectories by comparing the $\mathrm{CB}$ projection of a number of points that are uniformly distributed on a cubic Cartesian grid of cell side $\Delta_{c}$ within a cylinder of radius $r_{c}$ that is centered on the $t$-axis.

Let $m$ and $n$ be the indices for helical turns and X-ray source positions in each helical turn, respectively. For the $n$th X-ray source on the $m$ th helical turn, we can calculate two CB projections for any point by using the projection matrices from the first and second calibrated trajectories. Let $J$ be the total number of points whose projection is inside the detector, and let $\underline{c}_{j m n}$ be the $j$ th point. Let $\underline{c}_{1 j m n}^{\mathrm{p}}$ and $\underline{c}_{2 j m n}^{\mathrm{p}}$ be the projections of $\underline{c}_{j m n}$ using projection matrices from the first and second calibrated trajectories, respectively. As a figure-of-merit, we compute the root-of-mean-square error (RMSE) for the $n$th X-ray source on the $m$ th helical turn, denoted as $\sigma_{m n}$, using

$$
\sigma_{m n}^{2}=\frac{1}{J} \sum_{j=1}^{J}\left\|\underline{c}_{1 j m n}^{\mathrm{P}}-\underline{c}_{2 j m n}^{\mathrm{p}}\right\|^{2} .
$$

Here, $\underline{c}_{1 j m n}^{\mathrm{p}}$ and $\underline{c}_{2 j m n}^{\mathrm{p}}$ are expressed in the image coordinate system, so that $\sigma_{m n}$ is in detector pixel unit.

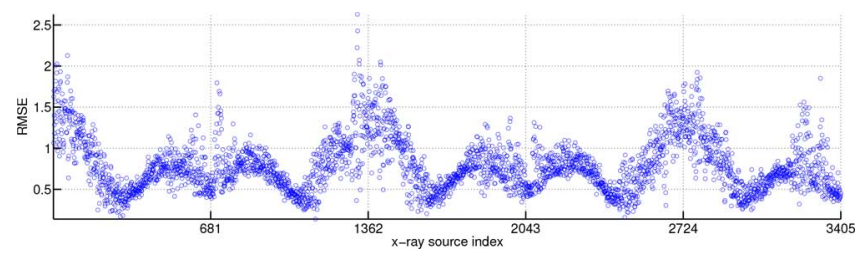

Fig. 6. RMSE measurement for experiment A using a virtual cylinder phantom that is of radius $r_{c}=130 \mathrm{~mm}$ and cell side $\Delta_{c}=10 \mathrm{~mm}$. Vertical axis is of detector pixel unit, i.e., $0.308 \mathrm{~mm} /$ pixel.

Note that other methods may be used for evaluating the repeatability of the reverse helical trajectory. For example, in [29], projection matrices are decomposed to obtain $\mathrm{CB}$ scan parameters, e.g., X-ray source positions, source-to-detector distances and detector orientations, and evaluations are performed for each individual parameter. However, these parameters are typically strongly correlated, so that errors present in each individual parameter may cancel each other to a certain extent when they are together employed to perform backprojection; the accuracy of back-projection directly impacts the quality of image reconstruction. Therefore, for our purpose, examining the similarity of projection of points is superior to examining that of each individual CB scan parameters. This approach was similarly used in [27].

We have conducted two experiments, A and B, on the same day. Each experiment consisted in performing two testing runs of the reverse helix with the PDS-3 phantom kept unchanged. From one experiment to the next, the phantom was taken out then replaced on the patient table. We computed the RMSE of (1) for each X-ray source position in both experiments using $r_{c}=130 \mathrm{~mm}$ (which is identical to the radius of the FOV) and $\Delta_{c}=10 \mathrm{~mm}$; altogether 25850 points were used. The RMSE of each X-ray source position for experiment A is shown in Fig. 6; a similar result was obtained for experiment $\mathrm{B}$. We also computed the mean and standard deviation (std) of RMSEs using all $\mathrm{X}$-ray source positions for both experiments. The mean and std of the RMSE of Experiment A are 0.79 and 0.32, respectively, whereas those of Experiment B are 0.87 and 0.27 , respectively. These results and Fig. 6 both demonstrate that an average accuracy of about 0.8 detector pixel (with pixel size of $0.308 \mathrm{~mm}$ ) can be achieved in the $\mathrm{CB}$ projections of the reverse helical trajectory. This accuracy indicates satisfactory repeatability of the reverse helical trajectory in the sense that, in average, the error is at sub-detector-pixel level.

\section{IMAGE RECONSTRUCTION}

This section describes our image reconstruction scheme, which consists of two steps: trajectory registration and the Fusion-RFDK method. Recall from Section III-B that the $t$-axis deviates from the rotational axis of the calibrated reverse helical trajectory. Similarly to circular CB imaging [19], such a deviation increases complexity in image reconstruction as well as regarding estimation of an appropriate FOV. Trajectory registration aims at reducing this complexity by transforming the calibrated reverse helix to another Cartesian coordinate system that is spanned by $(x, y, z)$ axes. Image reconstruction is performed in this $(x, y, z)$-coordinate system using our Fusion-RFDK method [15]. 


\section{A. Trajectory Registration}

Let $\left(\underline{e}_{x}, \underline{e}_{y}, \underline{e}_{z}\right)$ and $\underline{x}_{0}=\left(x_{0}, y_{0}, z_{0}\right)^{T}$ be the basis vectors and the origin of the $(x, y, z)$-coordinate system, respectively. The trajectory registration process aims at finding $\underline{e}_{x}, \underline{e}_{y}, \underline{e}_{z}$, and $\underline{x}_{0}$ such that the calibrated reverse helix in the $(x, y, z)$-coordinate system satisfies the following requirements as good as possible: 1) the rotational axis is the $z$-axis; 2) the translation of the reverse helix is along the $\underline{e}_{z}$ direction; 3) the first X-ray source position of the first helical turn lies on the $x$-axis. Vectors $\underline{e}_{x}, \underline{e}_{y}, \underline{e}_{z}$, and $\underline{x}_{0}$ are obtained as follows.

First, we explain how to get $\underline{e}_{z}, \underline{e}_{x}$, and $\underline{e}_{y}$. Let $\underline{a}_{m n}=$ $\left(r_{m n}, s_{m n}, t_{m n}\right)^{T}$ be the $(r, s, t)$ coordinates of the $n$th X-ray source position on the $m$ th helical turn. We define

$$
\underline{v}_{\alpha \beta n}=\left(r_{\alpha \beta n}, s_{\alpha \beta n}, t_{\alpha \beta n}\right)^{T}=\underline{a}_{\beta n}-\underline{a}_{\alpha n}
$$

where $\{\alpha, \beta\}=1, \ldots, M$, with $M$ being the total number of helical turns. By construction, if $\underline{a}_{\alpha n}$ and $\underline{a}_{\beta n}$ both belong to the right-handed or left-handed helical turns, vector $\underline{v}_{\alpha \beta n}$ may be expected to be parallel to the rotational axis. This observation indicates that the unit vector $\underline{e}_{z}$ can be obtained by

$$
\underline{e}_{z}=\underset{\left\{\underline{e}_{z}\right\}}{\arg \min } \sum_{(\alpha, \beta) \in \Psi} \sum_{n=1}^{N}\left\|\underline{v}_{\alpha \beta n} \times \underline{e}_{z}\right\|^{2}-\chi\left(\left\|\underline{e}_{z}\right\|^{2}-1\right)
$$

where $\Psi=\{(\alpha, \beta) \mid \alpha=1: M ; \beta=1: M ; \alpha \neq \beta ; \bmod (\alpha+$ $\beta, 2)=0\}$ with $\bmod (x, 2)$ being equal to $x$ modulo $2, \times$ is the operator for cross product, $\chi$ is a Lagrange multiplier, and $N$ is the total number of X-ray source positions per helical turn. The definition of $\Psi$ ensures that $\underline{a}_{\alpha n}$ and $\underline{a}_{\beta n}$ belong to the same type of helical turns (left- or right-handed), whereas the Lagrange multiplier makes sure that $\underline{e}_{z}$ is a unit vector. By taking the gradient of the expression on the right side of (2) with respect to $\underline{e}_{z}$ and setting the result to zero, we obtain $A \underline{e}_{z}=\chi \underline{e}_{z}$, where $A$ is expressed as

$$
\sum_{(\alpha, \beta) \in \Psi} \sum_{n=1}^{N}\left[\begin{array}{ccc}
s_{\alpha \beta n}^{2}+t_{\alpha \beta n}^{2} & -r_{\alpha \beta n} s_{\alpha \beta n} & -r_{\alpha \beta n} t_{\alpha \beta n} \\
-r_{\alpha \beta n} s_{\alpha \beta n} & r_{\alpha \beta n}^{2}+t_{\alpha \beta n}^{2} & -s_{\alpha \beta n} t_{\alpha \beta n} \\
-r_{\alpha \beta n} t_{\alpha \beta n} & -s_{\alpha \beta n} t_{\alpha \beta n} & r_{\alpha \beta n}^{2}+s_{\alpha \beta n}^{2}
\end{array}\right] .
$$

Therefore, $\underline{e}_{z}$ is an eigenvector of $A$.

Matrix $A$ is positive definite and symmetric, hence there are three eigenvectors of $A$. Note that the sign of an eigenvector is a degree of freedom. In this paper, we selected the eigenvector of $A$ that minimizes the right side of (2) as $\underline{e}_{z}$, and we chose the sign of $\underline{e}_{z}$ such that it points in the direction of translation of the reverse helix. Given $\underline{e}_{z}, \underline{e}_{x}$ and $\underline{e}_{y}$ are calculated using

$$
\underline{e}_{x}=\frac{\underline{a}_{11}-\left(\underline{a}_{11} \cdot \underline{e}_{z}\right) \underline{e}_{z}}{\left\|\underline{a}_{11}-\left(\underline{a}_{11} \cdot \underline{e}_{z}\right) \underline{e}_{z}\right\|}, \quad \underline{e}_{y}=\underline{e}_{z} \times \underline{e}_{x} .
$$

Now, we clarify how to obtain $\underline{x}_{0}=\left(x_{0}, y_{0}, z_{0}\right)^{T}$. Because $\underline{a}_{11}$ must lie on the $x$-axis, we have $z_{0}=\underline{a}_{11} \cdot \underline{e}_{z}$. If we assume the reverse helix is perfectly shaped with its rotational axis being parallel to the $t$-axis and through $\left(\hat{x}_{0}, \hat{y}_{0}, 0\right)^{T}$, then the distance

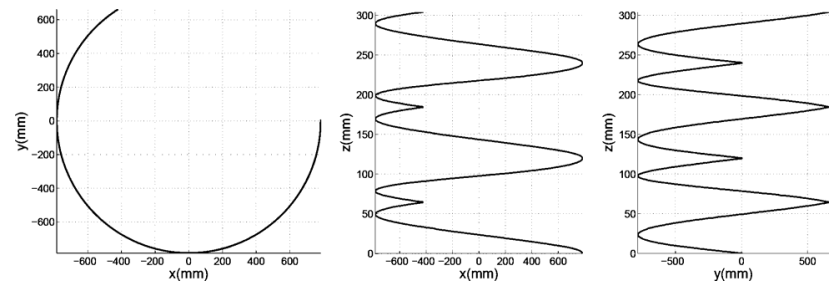

Fig. 7. Registered reverse helix, in the $(x, y, z)$-system. Left: orthogonal projection onto the $(x, y)$-plane; middle: onto the $(x, z)$-plane; right: onto the $(y, z)$-plane.

between X-ray source $\underline{a}_{m n}=\left(r_{m n}, s_{m n}, t_{m n}\right)^{T}$ and the rotational axis is equal to the rotational radius, which is denoted as $R$. Mathematically, it can be expressed as

$$
\left(\begin{array}{c}
\left(r_{11}-\hat{x}_{0}\right)^{2}+\left(s_{11}-\hat{y}_{0}\right)^{2} \\
\vdots \\
\left(r_{21}-\hat{x}_{0}\right)^{2}+\left(s_{21}-\hat{y}_{0}\right)^{2} \\
:
\end{array}\right)=\left(\begin{array}{c}
R^{2} \\
: \\
R^{2} \\
:
\end{array}\right)
$$

We estimate $\left(x_{0}, y_{0}\right)$ by solving the above equation in a least square sense.

Once $\underline{e}_{x}, \underline{e}_{y}, \underline{e}_{z}$, and $\underline{x}_{0}$ are obtained, trajectory registration can be achieved by transforming X-ray source positions and detector orientations from the world coordinate system to the $(x, y, z)$-system, as explained below. Let $\underline{a}$ be an X-ray source position in the world coordinate system, and let $\underline{b}=\left(b_{x}, b_{y}, b_{z}\right)^{T}$ be the corresponding coordinates of $\underline{a}$ in the $(x, y, z)$-system. Then $\underline{b}=Q_{1}\left(\underline{a}-\underline{x}_{0}\right)$, where $Q_{1}=\left(\underline{e}_{x}, \underline{e}_{y}, \underline{e}_{z}\right)^{T}$. Also, let $\left(\underline{e}_{u}^{\prime}, \underline{e}_{v}^{\prime}, \underline{e}_{w}^{\prime}\right)$ be the detector orientation after registration. Then, we have $\left(\underline{e}_{u}^{\prime}, \underline{e}_{v}^{\prime}, \underline{e}_{w}^{\prime}\right)=Q_{1}\left(\underline{e}_{u}, \underline{e}_{v}, \underline{e}_{w}\right)$. Note the source-to-detector distance and the detector center are not changed by the trajectory registration process as they are intrinsic parameters.

The registered trajectory meets all three requirements that were mentioned at the beginning of this subsection. See Fig. 7 for a graphical representation. Note from this figure how the registered trajectory is close to an ideal reverse helix unlike the unregistered trajectory.

We now analyze how close the registered reverse helix is to an ideal one. Let $\underline{b}_{x y}$ be the orthogonal projection of X-ray source $\underline{b}$ onto the $(x, y)$-plane. Let $\lambda$ be the azimuthal angle of $\underline{b}$, which is the counterclockwise polar angle of $\underline{b}_{x y}$ from the $x$-axis. Let $\Delta \lambda$ be the angular step size of $\lambda ; \Delta \lambda$ is positive for counterclockwise rotation and negative for clockwise rotation. Three observations can be made. First, the rotational radius is very close to the input value listed in Table I; the mean and standard deviation of $\left\|\underline{b}_{x y}\right\|$ are $783.94 \mathrm{~mm}$ and $0.41 \mathrm{~mm}$, respectively. Only small systematic deviations as well as statistical fluctuations are present; see the top row of Fig. 8. The systematic deviations may be caused by gravity, whereas the statistical fluctuations may be produced by small errors in the calibration process, such as inaccuracies in segmentation and modelization of the X-ray source focal spot as a single point. Second, the magnitude of the angular step size is in excellent agreement with the input value listed in Table I. The mean and standard deviation of $|\Delta \lambda|$ are $0.35^{\circ}$ and $0.01^{\circ}$, respectively; see the second row of Fig. 8 . Third, as expected, the $z$ coordinate increases linearly with the 

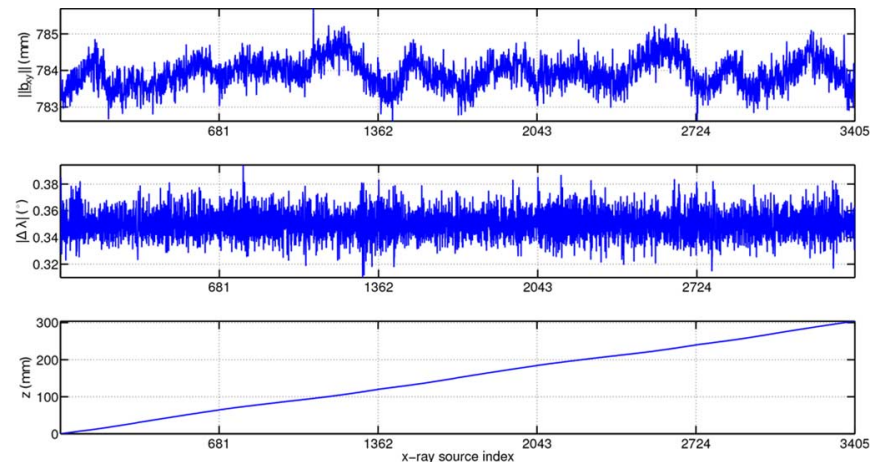

Fig. 8. Geometrical accuracy of the registered reverse helix. Top: rotational radius $(\mathrm{mm})$; middle: absolute angular increment $|\Delta \lambda|$ of X-ray source positions (degree); bottom: $z$ coordinates of X-ray source positions (mm).

source index as shown in the bottom row of Fig. 8. However, slight slope deviations can still be observed both within each helical turn and across consecutive helical turns. These deviations may be attributed to imperfection in our trajectory registration process. Indeed, we have observed in a numerical experiment that a deviation as small as $0.2^{\circ}$ in the polar angle of the rotational axis in spherical coordinate system can yield such deviations. This small deviation in the polar angle could also yield nonuniformity in the axial height of the helical turns. It was observed that the axial heights of the left-handed and right-handed helical turns are about 64 and $56 \mathrm{~mm}$, respectively; see Fig. 7. However, the registered trajectory was deemed suitable enough to define the FOV, and to perform image reconstruction using our Fusion-RFDK.

Based on the analysis above, we conclude that: 1) the trajectory registration process is practically satisfying; and 2) a close-to-ideal five-turn reverse helix can be practically implemented on a multiaxis $\mathrm{C}$-arm system.

\section{B. Fusion-RFDK}

The Fusion-RFDK method presented in [15] was only designed for two helical turns that were the same axial height. Thus, this method was not ready-to-use in our real data situation, because the registered trajectory consists of more than two turns and exhibits slightly different axial heights for different helical turns. An extension of the Fusion-RFDK method was created to accommodate these aspects. This extension is described hereafter.

We start the description of the Fusion-RFDK method by focussing on one basic cycle of the reverse helix, as illustrated in Fig. 9. This basic cycle is composed of one lower and one upper helical turn that are connected at the kink point $K$ of $z$ coordinate called $z_{\mathrm{K}}$. Let the axial heights of the lower and the upper helix be $H_{\mathrm{L}}$ and $H_{\mathrm{U}}$, respectively.

Also, let $\Omega_{\mathrm{L}}$ and $\Omega_{\mathrm{U}}$ be two volumes-of-interest (VOI) associated with the lower and the upper helical turns, respectively. Both $\Omega_{\mathrm{L}}$ and $\Omega_{\mathrm{U}}$ are cylinders of radius $r$ that are aligned on the $z$-axis and overlap in a region of axial height $H_{\mathrm{F}}$ that is centered on the kink plane $\left(z=z_{\mathrm{K}}\right)$. Let $z^{\prime}=z-z_{\mathrm{K}}$. Cylinder $\Omega_{\mathrm{L}}$ extends over $z^{\prime} \in\left[-H_{\mathrm{L}} / 2, H_{\mathrm{F}} / 2\right]$, whereas $\Omega_{\mathrm{U}}$ extends over $z^{\prime} \in\left[-H_{\mathrm{F}} / 2, H_{\mathrm{U}} / 2\right]$. The region of overlap, called the fusion zone, is denoted as $\Omega_{\mathrm{F}}=\Omega_{\mathrm{L}} \cap \Omega_{\mathrm{U}}$. The union of $\Omega_{\mathrm{L}}$

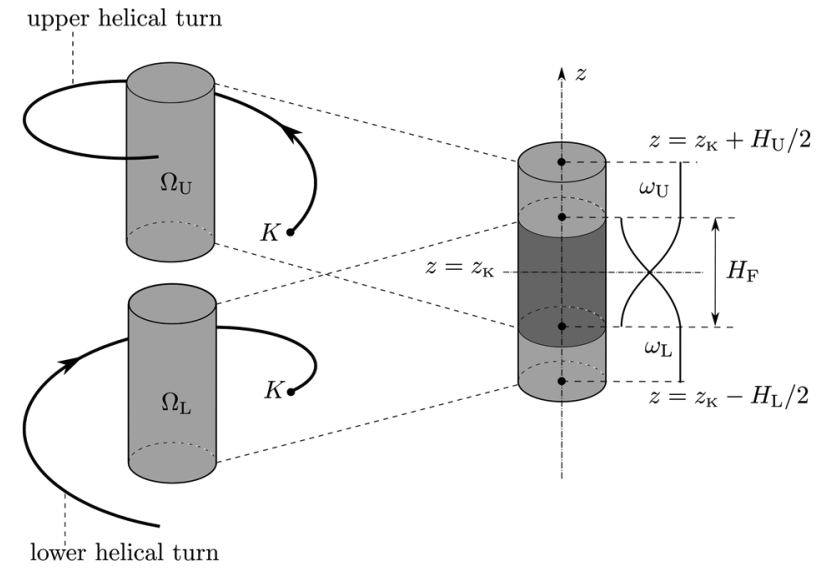

Fig. 9. Principles of Fusion-RFDK for one basic cycle. Lower (left-handed) and upper (right-handed) helical turns share the kink point $K$ with $z$ coordinate $z_{\mathrm{K}}$. Two volumes, denoted as $\Omega_{\mathrm{L}}$ and $\Omega_{\mathrm{U}}$, are reconstructed by the ramp-kernel based FDK algorithm using projection data from the lower and upper helical turns, respectively. These two volumes overlap each other in a fusion zone given by $z \in\left[z_{\mathrm{K}}-H_{\mathrm{F}} / 2, z_{\mathrm{K}}+H_{\mathrm{F}} / 2\right]$. In the fusion zone, $\Omega_{\mathrm{L}}$ and $\Omega_{\mathrm{U}}$ are combined by a fusion process using weighting functions $\omega_{\mathrm{L}}$ and $\omega_{\mathrm{U}}$.

and $\Omega_{\mathrm{U}}$ is called $\Omega_{\mathrm{C}}$, and this union is the region over which reconstruction is performed for the basic cycle. By construction, $H_{\mathrm{F}} \leq H_{\mathrm{L}}$ and $H_{\mathrm{F}} \leq H_{\mathrm{U}}$.

Reconstruction over $\Omega_{\mathrm{C}}$ goes as follows. First, we obtain two reconstructions, denoted as $f_{\mathrm{L}}(\underline{x})$ for $\underline{x} \in \Omega_{\mathrm{L}}$ and $f_{\mathrm{U}}(\underline{x})$ for $\underline{x} \in \Omega_{\mathrm{U}}$, by independently applying the ramp kernel based Feldkamp-Davis-Kress (RFDK) method [30], [31] to the lower and the upper helical turns, respectively. Second, we combine $f_{\mathrm{L}}(\underline{x})$ and $f_{\mathrm{U}}(\underline{x})$ by a fusion process using a pair of weighting functions. Let $f_{\mathrm{C}}(\underline{x})$ be the final image for $\underline{x} \in \Omega_{\mathrm{C}}$, then we have $f_{\mathrm{C}}(\underline{x})=\omega_{\mathrm{L}} f_{\mathrm{L}}(\underline{x})+\omega_{\mathrm{U}} f_{\mathrm{U}}(\underline{x})$, where

$$
\omega_{\mathrm{L}}\left(z^{\prime}\right)= \begin{cases}1 & z^{\prime} \in\left[-H_{\mathrm{L}} / 2,-H_{\mathrm{F}} / 2\right] \\ \cos ^{2}\left(\frac{z^{\prime} \pi}{2 H_{\mathrm{F}}}+\frac{\pi}{4}\right) & z^{\prime} \in\left[-H_{\mathrm{F}} / 2, H_{\mathrm{F}} / 2\right] \\ 0 & z^{\prime} \in\left[H_{\mathrm{F}} / 2, H_{\mathrm{U}} / 2\right]\end{cases}
$$

and $\omega_{\mathrm{U}}\left(z^{\prime}\right)=1-\omega_{\mathrm{L}}\left(z^{\prime}\right)$ for $z^{\prime} \in\left[-H_{\mathrm{L}} / 2, H_{\mathrm{U}} / 2\right]$.

Now, we discuss how reconstruction is performed when the reverse helix consists of several turns. The easiest way to understand the reconstruction concept is to picture the reverse helix as a sequence of successive cycles, each characterized by a label, $p$, that identifies the successive kink points (The first cycle consists of the first and second turns; the second cycle consists of the second and third turns; and so on). For each cycle, we have two volumes, now called $\Omega_{\mathrm{L}}^{p}$ and $\Omega_{\mathrm{U}}^{p}$ that overlap over a given fusion zone and define together a volume $\Omega_{\mathrm{C}}^{p}$. Reconstruction is performed over each volume $\Omega_{\mathrm{C}}^{p}$ using the equations above. By definition, the successive $\Omega_{\mathrm{C}}^{p}$ sets continuously form a long volume (with no gap and no overlap). This arrangement is depicted in Fig. 10 for cycles of index $p$ and $p+1$.

Note that a slightly different treatment was adopted for the first and last cycles. In the first cycle, the $z$ coverage for $\Omega_{\mathrm{L}}$ is extended so that the portion of $\Omega_{\mathrm{L}}$ below the kink point is $H_{\mathrm{L}} / 2+\left(H_{\mathrm{L}}-H_{\mathrm{F}}\right) / 2$ instead of $H_{\mathrm{L}} / 2$. In the last cycle, the $z$ coverage for $\Omega_{\mathrm{U}}$ is extended so that the portion of $\Omega_{\mathrm{U}}$ above the kink point is $H_{\mathrm{U}} / 2+\left(H_{\mathrm{U}}-H_{\mathrm{F}}\right) / 2$ instead of $H_{\mathrm{U}} / 2$. This change was introduced so as to better use the data available over 


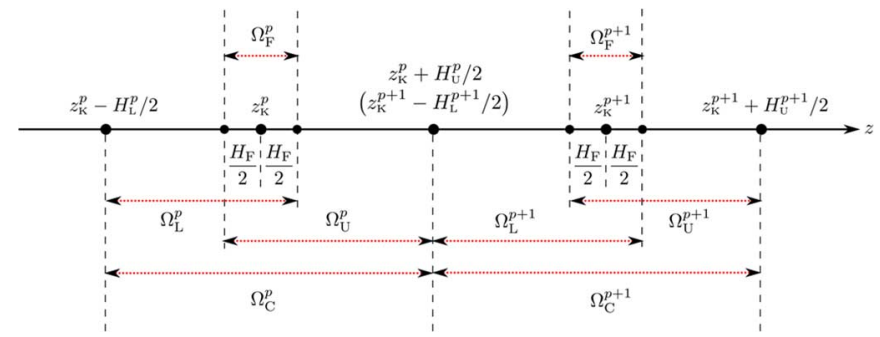

Fig. 10. Illustration of volumes involved in reconstruction from two successive cycles, of index $p$ and $p+1$. In particular, notice how $\Omega_{\mathrm{C}}^{p}$ and $\Omega_{\mathrm{C}}^{p+1}$ complement each other to form a long volume.

the first and last helical turns while not allowing reconstruction within a region of length $H_{\mathrm{F}}$ centered on each endpoint of the reverse helix, where noticeable $\mathrm{CB}$ artifacts are expected [16].

Aside from illustrating how volumes from subsequent cycles form together a long volume, Fig. 10 also shows the volume over which the RFDK method needs to be applied for each turn of the helix, except the first and the last turn. This volume starts at a distance $H_{\mathrm{F}} / 2$ before the first point on the turn and ends at a distance $H_{\mathrm{F}} / 2$ after the last point on the turn. In other words, it is centered on the midpoint of the helical turn (that is to say in $z$ ) and is of length equal to the axial length of the helical turn plus $H_{\mathrm{F}}$. For the first and last helical turns, the volume is of length equal to the axial length of the helical turn. According to the above description and Table I, the length of the volume covered in our experiment is expected to be: $\left(5 \times 60-H_{\mathrm{F}}\right) \mathrm{mm}$.

\section{Practical Issues}

Four aspects deserve special attention when implementing the Fusion-RFDK method for real data. First, for each basic cycle, the kink plane needs to be specified. By definition, the kink plane is perpendicular to the $z$-axis and goes through the kink point. However, this kink point will usually not exist due to finite sampling. In this paper, we define the $z$ coordinate of the kink point as the average of the $z$ coordinates of the last $\mathrm{X}$-ray source position of the lower helical turn and the first X-ray source position of the upper helical turn.

Second, the detector size imposes constraints on the distance covered by each helical turn, as well as $H_{\mathrm{F}}$. Let $H_{\mathrm{d}}$ be the height of the detector along the $z$-axis, and let $H_{\max }$ be the largest distance covered over one helical turn (recall that we are allowing the helical turns to cover different distances). To make sure that sufficient projection data are available for image reconstruction, $H_{\max }$ and $H_{\mathrm{F}}$ have to satisfy the following relation (with $\rho$ being the radius of the $\mathrm{ROI}$ )

$$
H_{\mathrm{F}}+2 H_{\max } \leq H_{\mathrm{d}}(R-\rho) / D .
$$

Third, the backprojection step in the RFDK method should be carefully implemented, because the increment in $\lambda$ varies from view to view. We have included this nonconstant angular increment in the back-projection step using the trapezoid rule.

Fourth, we adjusted the Parker-like weighting function as follows. Consider a helical turn and a line diverging from one source position on this turn. First, we orthogonally project this line and the helical turn onto the $(x, y)$-plane and obtain a line $\mathcal{L}$ and curve $\mathcal{C}$ in this plane. Because the angular length of each
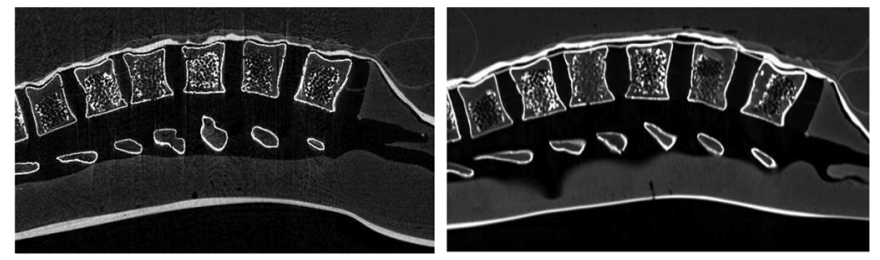

Fig. 11. Left: reconstructions of the torso phantom using the reverse helica data from the multiaxis C-arm system; voxel size: $0.5 \mathrm{~mm} \times 0.5 \mathrm{~mm} \times 0.5 \mathrm{~mm}$. Right: reconstructions from a state-of-the-art CT scanner; voxel size: 0.791 $\mathrm{mm} \times 0.791 \mathrm{~mm} \times 0.3 \mathrm{~mm}$. CT image on the right was linearly transformed such that its CT numbers for foam and air are equivalent to those of the reverse helix image on the left. Axial length: $274 \mathrm{~mm}$; display window: [ 1000,640$]$ HU.

helical turn is less than $360^{\circ}$, there are at most two intersections between $\mathcal{L}$ and $\mathcal{C}$. If $\mathcal{L}$ has one intersection with $\mathcal{C}$, we set the weight to 1 . If there are two intersections, one of them is the projection of the current source, and the other one intersects $\mathcal{C}$ between the projection of two other sources. The angular position of this intersection can be computed by linear interpolation and considered as the complementary angle of the projection of the current source position.

\section{RESUlTS}

In this section, we first present image reconstruction results from the reverse helical trajectory, comparing them either with results from a CT scan or against a graphical depiction of the ground truth, depending on the phantom. The purpose of this comparison is to assess anatomical/structural accuracy as well as getting acquainted with the visual appearance of reverse helix results. Next, a more quantitative comparison is performed by benchmarking the reverse helix results against those obtained with a classical circular short scan.

\section{A. Extended-Volume Reconstruction Results}

Reconstructions of voxel size $0.5 \mathrm{~mm} \times 0.5 \mathrm{~mm} \times 0.5 \mathrm{~mm}$ and $0.2 \mathrm{~mm} \times 0.2 \mathrm{~mm} \times 0.2 \mathrm{~mm}$ were obtained for the torso and CATSCB phantoms, respectively; see Figs. 11 (left) and 12 (bottom row). For these reconstructions, the ramp filter was applied with a Hamming window, and a fusion length of $H_{\mathrm{F}}=$ $30 \mathrm{~mm}$ was used.

The two reconstructions are $274 \mathrm{~mm}$ long. They demonstrate a smooth variation of attenuation coefficients along the axial direction with no pattern that can be easily related to geometrical aspects of the trajectory such as the kink points. Slight CB artifacts can be noticed in Fig. 11, which is not surprising since the Fusion-RFDK algorithm is an approximate method. However, no abrupt changes due to the nature of the data acquisition are observed in these $\mathrm{CB}$ artifacts.

Both reconstruction results demonstrate high anatomical-/structural accuracy. The anatomical appearance of the torso phantom was validated using reference images obtained from a state-of-the-art medical CT scanner (SOMATOM Definition, Siemens AG, Healthcare, Forchheim, Germany); see Fig. 11 (right). Note that a linear transformation was applied to the CT image such that the CT numbers for foam and air are, in average, equivalent to those in the reverse helix image; this transformation was needed due to differences in X-ray source 


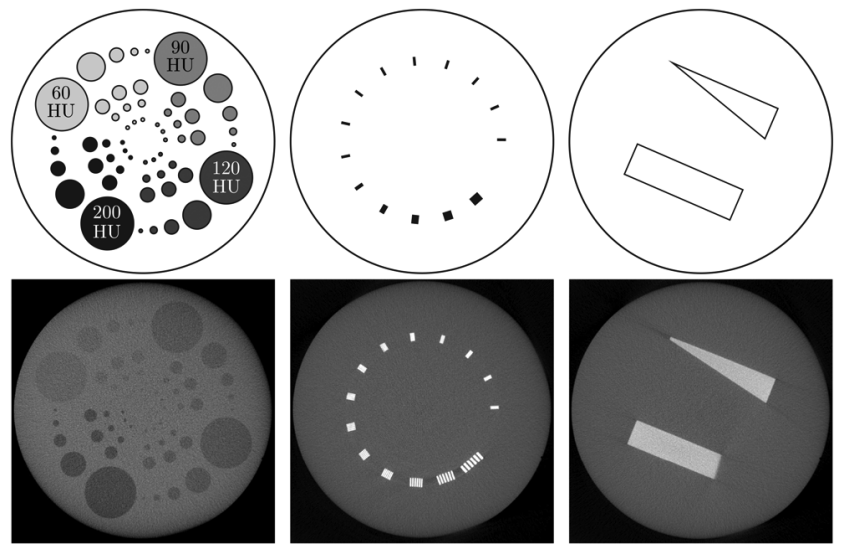

Fig. 12. Top row: ground truth, as provided by QRM (Möhrendorf, Germany). Bottom row: reconstruction results of the Siemens CB phantom from the reverse helical trajectory. Left to right: contrast pattern, resolution pattern and MTF edge pattern. Voxel size: $0.2 \mathrm{~mm} \times 0.2 \mathrm{~mm} \times 0.2 \mathrm{~mm}$; display window from left to right: $[-500,1000] \mathrm{HU},[-1000,2000] \mathrm{HU}$ and $[-1000,2000] \mathrm{HU}$.

spectra. Slight misalignment can be observed between these two images, because the two images are not registered. Also, the position of the SAWBONES spine relative to the torso foam slightly changed between the two scans, due to the foam being only loosely attached to the spine. This change in relative position explains the strong shading below the second vertebra from the left in Fig. 11 (right). Except for these differences, our reconstruction results are highly consistent with the $\mathrm{CT}$ images.

The structural accuracy of the CATSCB phantom was verified using the ground truth provided by QRM [32]. Fig. 12 demonstrates that our reconstruction results are in good agreement with the ground truth for this phantom as well. There are three contrast patterns in the Siemens CB phantom, Fig. 12 shows the one that has the following four contrast steps: $60 \mathrm{HU}, 90 \mathrm{HU}$, $120 \mathrm{HU}$, and $200 \mathrm{HU}$. Note that these contrast steps are defined for $120 \mathrm{kVp}$, and the Siemens CB phantom in our experiment was scanned at $91.2 \mathrm{kVp}$. Hence, the contrast steps shown in the bottom left of Fig. 12 maybe slightly different from the reference numbers.

\section{B. Comparison With Circular CB Imaging}

In this section, the reconstructions from the reverse helical trajectory are compared to results obtained from a circular shortscan, both by visual inspections and using quantitative metrics. The primary purpose of this comparison is to verify that the longer axial coverage of the reverse helix are acceptable in comparison with images used in clinical routine.

The circular trajectory was implemented on the same multiaxis $\mathrm{C}$-arm system. It consists of $638 \mathrm{X}$-ray source positions with an angular step size of $0.37^{\circ}$, covering a total angular length of $236.06^{\circ}$. The torso phantom was scanned using $(90 \mathrm{kVp}, 43 \mathrm{~mA})$ with average pulsewidth $3.1 \mathrm{~ms}$, whereas the CATSCB phantom was scanned using $(90 \mathrm{kVp}, 137 \mathrm{~mA})$ with average pulsewidth $6.3 \mathrm{~ms}$. The dose-area-product measurements for the torso and CATSCB phantom were 60.451 $\mathrm{mGy} \cdot \mathrm{cm}^{2}$ and $304.961 \mathrm{mGy} \cdot \mathrm{cm}^{2}$, respectively. Note that these numbers are comparable but not exactly the same as those used for each turn on the reverse helix. These slight differences are due to utilization of predefined protocols.
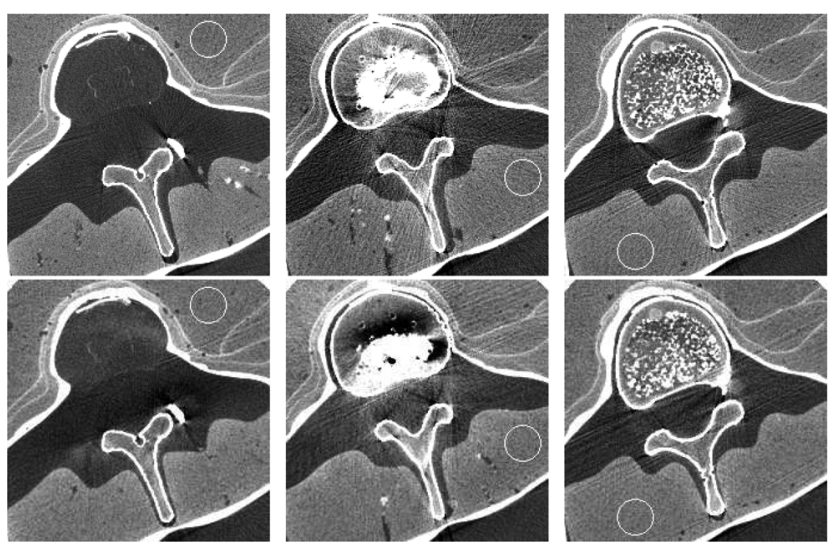

Fig. 13. Reconstructions of the torso phantom. Top row: results from the reverse helical trajectory; the slice distance to the kink plane is, from left to right, $0 \mathrm{~mm}, 30 \mathrm{~mm}$, and $60 \mathrm{~mm}$. Bottom row: results from the circular trajectory; the slice distance to the circular plane is, from left to right, $60 \mathrm{~mm}, 30 \mathrm{~mm}$, and $0 \mathrm{~mm}$. Voxel size: $0.5 \mathrm{~mm} \times 0.5 \mathrm{~mm} \times 0.5 \mathrm{~mm}$; display window: $\mu_{\text {anchor }}+$ $[-500,500]$ HU. Display anchor $\mu_{\text {anchor }}$ was calculated individually for each slice using pixels within the indicated circles.

Circular CB data were acquired for both the torso phantom and the CATSCB phantom. Image reconstruction was performed using the RFDK method with Hamming apodization of the ramp filter. Two $160-\mathrm{mm}$-long volumes of voxel size 0.5 $\mathrm{mm} \times 0.5 \mathrm{~mm} \times 0.5 \mathrm{~mm}$ and $0.2 \mathrm{~mm} \times 0.2 \mathrm{~mm} \times 0.2 \mathrm{~mm}$ were reconstructed for the torso and CATSCB phantom, respectively. These volumes were centered on the trajectory plane. Because the circular and reverse helical data acquisitions involved different angular positions for the first source position when scanning the torso phantom, registration was needed to compare the reconstructions of the torso phantom. This registration was achieved manually using bilinear interpolation. Due to this interpolation, the circular $\mathrm{CB}$ reconstructions of the torso phantoms may appear slightly smoother. For the CATSCB phantom, the data acquisitions involved the same angular position for the first source position, so that the reconstructed volumes were close to each other and registration was deemed not necessary.

We start the comparison with a visual inspection. Slight lowfrequency differences were observed between the results from the reverse helical trajectory and those from the circular trajectory. These differences were seen as a combination of $\mathrm{CB}$ artifacts and residual errors from physical effects. To account for these low-frequency differences in image display, we introduced a display anchor called $\mu_{\text {anchor }}$. For each slice, we select a homogeneous region and calculate the mean of the CT number in this region. This mean value is the display anchor and is used as a reference point for the display window. For example, consider the first column of Fig. 13. First, we select two equivalent homogeneous regions in these two images, which are indicated by the two circles. Then we individually calculate a display anchor, $\mu_{\text {anchor }}$, for each image using pixels in this region and set the display window to $\mu_{\text {anchor }}+[-500,500]$. In this way, the grayscale values of the two images being compared are brought to the same level.

Different observations were made between the transverse and axial directions. In the transverse direction, the reverse helix images were in general comparable to those from the 


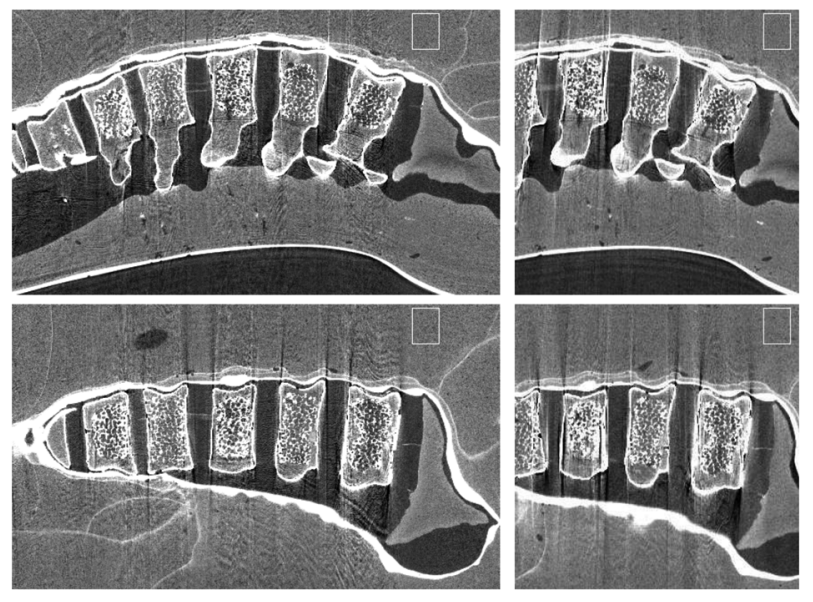

Fig. 14. Reconstructions of the torso phantom. Top: sagittal view; bottom: coronal view. Left: $274 \mathrm{~mm}$ images from the reverse helix; right: $160 \mathrm{~mm}$ images from the circular trajectory. Voxel size: $0.5 \mathrm{~mm} \times 0.5 \mathrm{~mm} \times 0.5 \mathrm{~mm}$; display window: $\mu_{\text {anchor }}+[-500,500] \mathrm{HU}$. The display anchor $\mu_{\text {anchor }}$ was calculated individually for each slice using pixels within the indicated white boxes.

circular trajectory. One trajectory may outperform the other in some regions, but overall the performance was fairly similar; see Fig. 13. In the axial direction, CB artifacts significantly vary with slice position for the circular trajectory, whereas they appear more uniform and less noticeable for the reverse helix; see Fig. 14. Given the properties of FDK reconstruction with a circular trajectory, this difference is not surprising. Indeed, for the circular trajectory, Tuy's condition is only satisfied in the trajectory plane; the further a slice is away from the trajectory plane, the less complete the data is for image reconstruction and the stronger $\mathrm{CB}$ artifacts can be. In contrast, the reverse helix satisfies Tuy's condition everywhere within its convex hull and provides projection data evenly along the axial direction. This advantageous feature of the reverse helix helps both reducing and uniformly distributing $\mathrm{CB}$ artifacts along the axial direction, as far as utilization of Fusion-RFDK is concerned. If the reconstruction algorithm had been exact, $\mathrm{CB}$ artifacts could have been fully avoided. Pearson et al. [7] nicely illustrated this aspect using real reverse helical data versus circular data.

Now, the results from the two source trajectories are quantitatively compared by using four sections of the Siemens CB phantom [32]: Section G (plain), Section A (contrast), Section $\mathrm{D}$ (spatial resolution), and Section F (MTF edge). First, we study uniformity using the plain section. We averaged 10 slices within the plain section for both the reverse helix and the circular trajectory; these averages are shown in the top row of Fig. 15. Capping artifacts are observed in both images. These capping artifacts appear mainly near the boundary of the phantom and vary according to position on this boundary; see the profiles in the middle and bottom rows of Fig. 15. The capping artifact is stronger in the image from the reverse helix than in that from the circular trajectory, possibly because the data correction methods used in our experiment are optimized for circular CB images, not for reverse helical images.

Second, contrast-to-noise ratio (CNR) was investigated using the contrast pattern. Recall from Fig. 12 that the contrast pattern has four different contrast steps. We call the linear attenuation
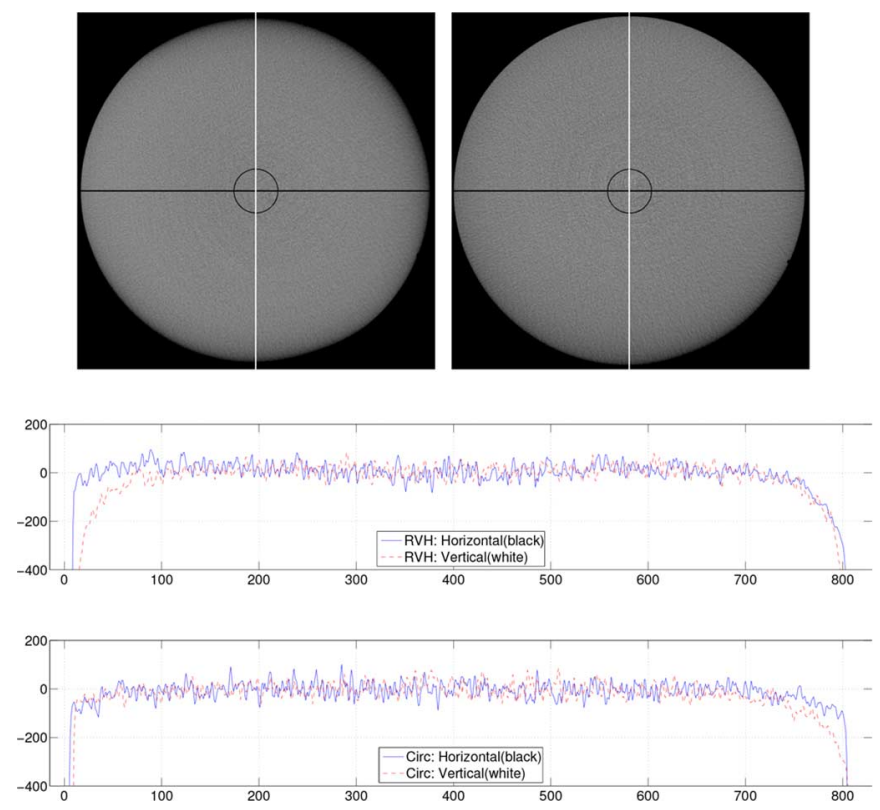

Fig. 15. Average of 10 slices within the plain section (Section G) of the Siemens CB phantom. Top left: result from the reverse helical trajectory; top right: result from the circular trajectory. Voxel size: 0.2 $\mathrm{mm} \times 0.2 \mathrm{~mm} \times 0.2 \mathrm{~mm}$; display window: $\mu_{\text {anchor }}+[-500,500] \mathrm{HU}$. The display anchor, $\mu_{\text {anchor }}$, was calculated individually for each image using pixels within the centered black circle. Middle and bottom rows show the profiles of the top left and right images, respectively. Each profile was subtracted by the corresponding $\mu_{\text {anchor }}$. Vertical axes: HU; horizontal axes: pixel index.
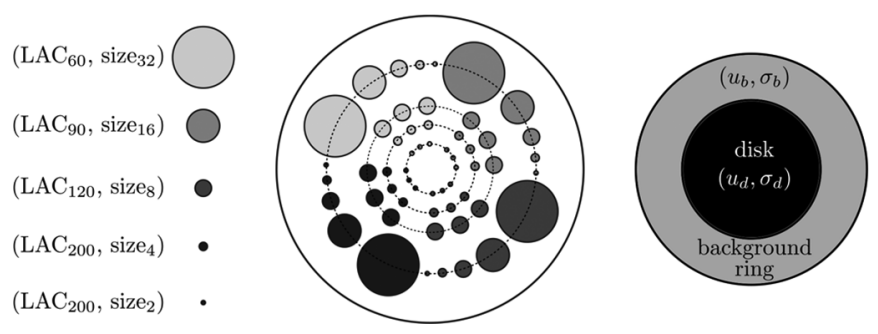

Fig. 16. Contrast pattern (Section A) of the Siemens CB phantom. Left: terminology for LAC and size; middle: the contrast pattern; right: illustration for CNR calculation.

coefficients (LACs) for the contrast of $60 \mathrm{HU}, 90 \mathrm{HU}, 120 \mathrm{HU}$, and $200 \mathrm{HU}$ as $\mathrm{LAC}_{60}, \mathrm{LAC}_{90}, \mathrm{LAC}_{120}$, and $\mathrm{LAC}_{200}$, respectively; see Fig. 16. For each LAC, there are disks of five different diameters: $2 \mathrm{~mm}, 4 \mathrm{~mm}, 8 \mathrm{~mm}, 16 \mathrm{~mm}$, and $32 \mathrm{~mm}$; see [32]. We refer to these disks as being of size $_{2}$, size $_{4}$, size $_{8}$, size $_{16}$, and size $_{32}$, respectively.

The disks are on four concentric circles; see the dashed circles in Fig. 16. For CNR measurement, we selected the disks of size 8 and size $_{4}$ that are located on the middle two circles. The disks on the outermost circle were not included due to overlap with capping artifacts, whereas the disks on the innermost circle were not used due to poor detectability.

For each selected circle, three disks of the same LAC and the same size form a cluster. For example, in Fig. 16, the three black disks located on the second outermost dashed circle can be identified as $\left(\mathrm{LAC}_{200}, \mathrm{size}_{8}\right)$. For each cluster, we first calculate the CNR of each disk, then compute the average of all three CNRs as the final CNR of the cluster. Fig. 16 illustrates how 
TABLE III

CNR COMPARISON BETWEen THE REVERSE HeliCAL TRAJECTORY (LEFT: RVH) AND THE CIRCULAR TRAJECTORY (RIGHT: CiRC) USING THE Contrast Pattern (Section A of the Siemens CB Phantom)

\begin{tabular}{c|c|c|c|c|c|c|c|c}
\hline \hline RVH & Circ & \multicolumn{2}{|c|}{ LAC $_{60}$} & \multicolumn{2}{c|}{ LAC $_{90}$} & \multicolumn{2}{c|}{ LAC $_{120}$} & \multicolumn{2}{c}{ LAC $_{200}$} \\
\hline size $_{8}$ & 0.93 & 0.83 & 1.52 & 1.36 & 2.00 & 1.81 & 2.87 & 2.67 \\
\hline size $_{4}$ & 0.91 & 0.75 & 1.29 & 1.28 & 1.73 & 1.61 & 2.71 & 2.34 \\
\hline \hline
\end{tabular}
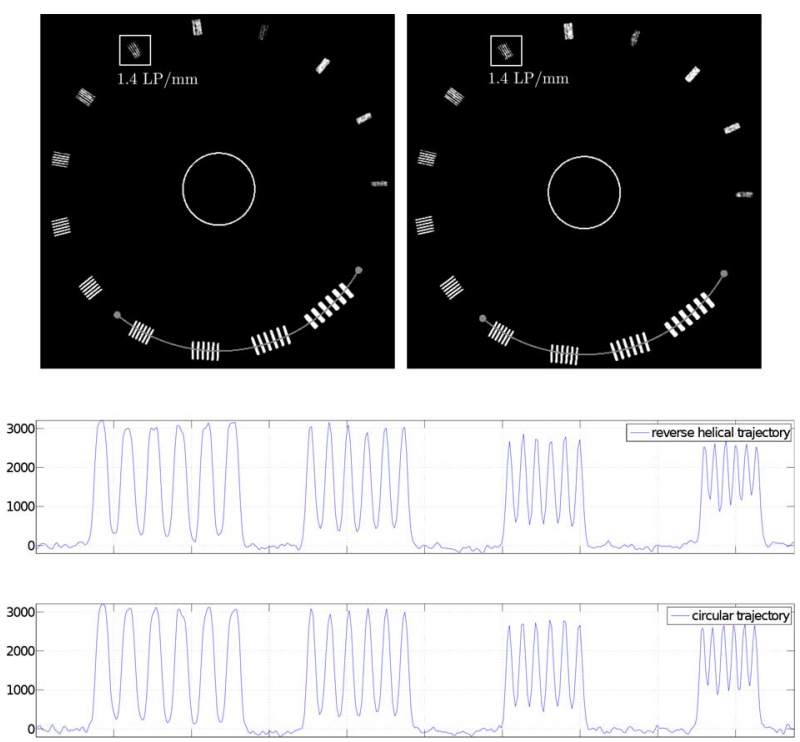

Fig. 17. Results of the resolution pattern (Section D) of the Siemens CB phantom. Top left: image result from the reverse helical trajectory; top right: image result from the circular trajectory. Voxel size: $0.2 \mathrm{~mm} \times 0.2 \mathrm{~mm} \times 0.2$ $\mathrm{mm}$; display window: $\mu_{\text {anchor }}+[1500,1900] \mathrm{HU}$. The display anchor $\mu_{\text {anchor }}$ was calculated individually for each image using pixels within the centered white circle. Middle and bottom rows show the profiles along the gray circular curve segments of the top left and right images, respectively. Each profile was subtracted by the corresponding $\mu_{\text {anchor }}$.

the CNR was computed for any given disk. Let $\mu_{\mathrm{d}}$ and $\sigma_{\mathrm{d}}$ be the mean and standard deviation of the LAC of the pixels inside the disk (black), respectively. Let $\mu_{\mathrm{b}}$ and $\sigma_{\mathrm{b}}$ be the mean and standard deviation of the LAC of the background pixels (gray ring), respectively. The CNR of the disk was defined by

$$
\mathrm{CNR}=\left|\mu_{\mathrm{b}}-\mu_{\mathrm{d}}\right| / \sqrt{\left(\sigma_{\mathrm{b}}^{2}+\sigma_{\mathrm{d}}^{2}\right) / 2} .
$$

The CNR results are listed in Table III. As expected, for the same LAC, the CNR of size 8 is higher than that of size 4 . Also, note that the reconstruction result used for Table III was not in the fusion zone, thus it only used projection data from one single helical turn. Given that the exposure associated with one helical turn was comparable to that associated with the circular trajectory, Table III indicates that the two trajectories produced images of comparable contrast.

Finally, spatial resolution was examined using both the resolution and the MTF edge patterns. From a visual inspection, the results from both source trajectories clearly have comparable spatial resolution; see Fig. 17. The profiles along the white curve segments in both images are very similar to each other. According to the data sheet of the Siemens CB phantom [32], both images have spatial resolution around $1.4 \mathrm{LP} / \mathrm{mm}$.

The modulation transfer function (MTF) was computed using the edge spread function corresponding to the edge indicated

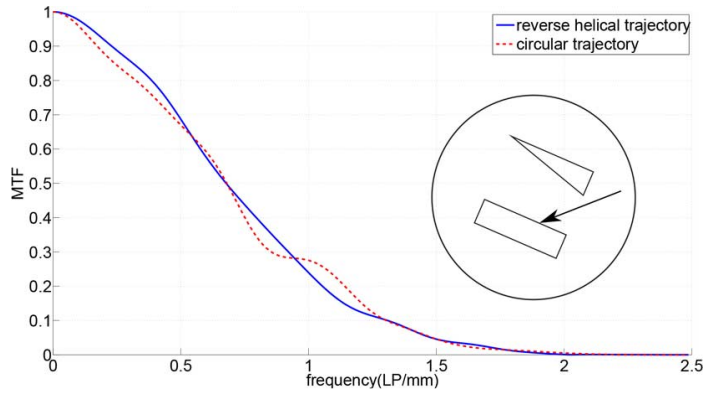

Fig. 18. Resolution assessment using the edge pattern (Section $F$ of the Siemens CB phantom). MTF curves were measured using the edge indicated by the arrow.

by the arrow in Fig. 18. The resulting MTF curves in Fig. 18 are very close to each other. Note that the maximum detectable spatial frequencies (corresponding to the $0.1 \mathrm{MTF}$ value) for the two MTF curves are both around $1.3 \mathrm{LP} / \mathrm{mm}$, which is in agreement with the observations from Fig. 17. Hence, both visual inspections and quantitative measurements suggest that the spatial resolution for the reverse helix is comparable to that for the circular trajectory.

\section{DiscuSSION AND CONCLUSION}

We have successfully demonstrated that long FOV imaging using a reverse helix is feasible in the interventional room. This demonstration involved developing a novel calibration method, assessing repeatability, implementing a reconstruction method, and quantitatively evaluating image quality.

In our demonstration, the reverse helix was realized without moving the patient table by using the motion flexibility offered by a multiaxis $\mathrm{C}$-arm system. Using translation of the patient table together with a rotation of the $\mathrm{C}$-arm would have been another approach. However, moving the patient may not be practical, particularly in the middle of a surgical intervention. Moreover, the patient table in the interventional room is a floating table that is not designed to allow precise motion control in the same way as a CT table is. Thus, using patient table translation may not be possible.

The reverse helix that we implemented consisted of five turns of $240^{\circ}$ covering each an axial distance of $60 \mathrm{~mm}$. This five-turn reverse helix was successfully calibrated using a novel method, which is an extension of the approach used in [22] and [23]. The extension involved introducing a new calibration phantom, the PDS-3 phantom, that is $450 \mathrm{~mm}$ long and uses a 10-bit encoding scheme. This phantom is sufficient to calibrate as many as seven turns of $60 \mathrm{~mm}$. For a longer coverage, this phantom could simply be extended using more balls. We have also demonstrated that the five-turn reverse helix can be in average repeated with subdetector-pixel accuracy. However, this repeatability was only examined within a short period of time (less than $2 \mathrm{~h}$ ). Repeatability within a much longer period of time, such as within one year, will be investigated in the future as it is of great importance for clinical practice. For circular CB scans, the trajectory is typically calibrated only once a year. Note also that repeatability was only investigated for the $2 \times 2$ binning mode.

Image reconstruction was performed using our Fusion-RFDK method with several adaptations that allowed accounting for 
slight geometrical deviations. High anatomical and structural accuracy was demonstrated for two phantoms. Moreover, a quantitative comparison against a short circular scan was performed. The primary purpose of this comparison was to verify that the extended coverage is achieved with satisfactory image quality. Except in the fusion zones, the Fusion-RFDK method performed reconstruction at each point using the same amount of projections as the short circular scan; in the fusion zone, the amount was double. Results from the reverse helix demonstrated comparable spatial resolution (about 1.3 $\mathrm{LP} / \mathrm{mm}$ ); milder CB artifacts; and also comparable CNR in a region outside the fusion zones, where similar X-ray exposure is used for reconstruction. However, slightly more pronounced capping artifacts were observed with the reverse helix. Further investigations are needed to identify how to better mitigate these capping artifacts.

Because the reverse helix provides complete data, reconstruction with no $\mathrm{CB}$ artifacts could have been demonstrated by using an exact algorithm rather than the Fusion-RFDK method. Such a demonstration was not the focus of this paper, particularly as the data completeness advantage of the reverse helix over the circular scan has already been nicely demonstrated with real data by Pearson et al. [7]. However, we plan to apply various exact algorithms to our data in the future and compare their performance.

In our comparison, the short circular scan provided a 160-mm-long volume whereas the five-turn reverse helix yielded a 274-mm-long volume. In both cases, the radius of the reconstructed volume was $130 \mathrm{~mm}$, which corresponds to using the detector in the landscape mode. Thus, two short parallel circular scans would have provided a little more coverage with 2.5 times less exposure, but these advantages would be at the cost of $\mathrm{CB}$ artifact and noncontinuous data acquisition. Continuity of the trajectory is known to be a key ingredient for mitigation of motion artifacts, which has been used extensively in CT (e.g., [33]). If the comparison was done with full parallel circular scans (which reduces the strength of $\mathrm{CB}$ artifacts) and if the reverse helix was implemented with rotations of $200^{\circ}$ and a pitch of $80 \mathrm{~mm}$, four turns on the reverse helix would provide the same axial coverage as two full scans with only $11 \%$ more exposure. In this comparison, the reverse helix would allow continuous data acquisition and yield complete data, with $400^{\circ}$ of data for each axial slice. Conservative parameters $\left(240^{\circ}\right.$ and $60 \mathrm{~mm}$ ) were used in our implementation of the reverse helix because we did not know ahead of time how accurately the reverse helix could be implemented. Also, we would like to point out that there is a lot of unexplored features of the reverse helix acquisition in terms of angular coverage per turn, utilization of data redundancies and beam collimation. For example, for imaging the carotid artery from the aortic arch to the brain, the multiaxis $\mathrm{C}$-arm system that we used in this paper allows the angular coverage per turn to be as high as $400^{\circ}$, which brings richness in terms of image reconstruction.

Technically speaking, there is still a large body of knowledge that needs to be built to identify what is the best way to perform long-object imaging in the interventional room. First, further image reconstruction algorithm developments could be beneficial for the reverse helix, particularly in terms of data require- ments. Second, the reverse helix is not the only complete trajectory that can be implemented for long-object imaging using a multiaxis $\mathrm{C}$-arm system. Comparison with other trajectories [34], [35], including a set of parallel circular scans, is of high interest to us and will be considered in the future. Although this paper was focused on the reverse helix, the knowledge that was acquired gives us strong optimism that other trajectories can be as robustly implemented.

\section{ACKNOWLEDGMENT}

The authors would like thank the reviewers for pointing out [7].

\section{REFERENCES}

[1] C. A. Binkert, H. Alencar, J. Singh, and R. A. Baum, "Translumbar type II endoleak repair using angiographic CT," J. Vasc. Interv. Radiol., vol. 17, no. 8, pp. 1349-1353, Aug. 2006.

[2] R. Hodek-Wuerz, J.-B. Martin, K. Wilhelm, K. O. Lovblad, D. Babic, D. A. Rufenacht, and S. G. Wetzel, "Percutaneous vertebroplasty: Preliminary experiences with rotational acquisitions and 3-D reconstructions for therapy control," Cardiovasc. Interv. Rad., vol. 29, no. 5, pp. 862-865, Sep./Oct. 2006.

[3] S. Hirota, N. Nakao, S. Yamamoto, K. Kobayashi, H. Maeda, R. Ishikura, K. Miura, K. Sakamoto, K. Ueda, and R. Baba, "Cone-beam CT with flat-panel-detector digital angiography system: Early experience in abdominal interventional procedures," Cardiovasc. Interv. Rad., vol. 29, no. 6, pp. 1034-1038, Nov./Dec. 2006.

[4] B. C. Meyer, B. B. Frericks, T. Albrecht, K.-J. Wolf, and F. K. Wacker, "Contrast-enhanced abdominal angiographic CT for intra-abdominal tumor embolization: A new tool for vessel and soft tissue visualization," Cardiovasc. Interv. Rad., vol. 30, no. 4, pp. 743-749, Jul. 2007.

[5] M. J. Wallace et al., "Impact of C-arm CT on hepatic arterial interventions for hepatic malignancies," J. Vasc. Interv. Radiol., vol. 18, no. 12, pp. 1500-1507, Dec. 2007.

[6] M. J. Wallace, M. D. Kuo, C. Glaiberman, C. A. Binkert, R. C. Orth, and G. Soulez, "Three-dimensional C-arm cone-beam CT: Applications in the interventional suite," J. Vasc. Interv. Radiol., vol. 19, no. 6, pp. 799-813, Jun. 2008.

[7] E. Pearson, S. Cho, C. A. Pelizzari, and X. Pan, "Non-circular cone beam CT trajectories: A preliminary investigation on a clinical scanner," in Proc. Nucl. Sci. Symp. Med. Imag. Conf. Rec., 2010, pp. 3172-3175.

[8] F. Dennerlein and F. Noo, "Cone-beam artifact evaluation of the factorization method,” Med. Phys., vol. 38, no. S1, pp. S18-S24, 2011.

[9] J. Tan, H. H. Li, E. Klein, H. Li, P. Parikh, and D. Yang, "Physical phantom studies of helical cone-beam CT with exact reconstruction," Med. Phys., vol. 39, no. 8, pp. 4695-4704, 2012.

[10] S. Cho, D. Xia, C. A. Pelizzari, and X. Pan, "Exact reconstruction of volumetric images in reverse helical cone-beam CT," Med. Phys., vol. 35, no. 7, pp. 3030-3040, Jul. 2008.

[11] H. K. Tuy, "An inversion formula for cone-beam reconstruction," SIAM J. Appl. Math., vol. 43, no. 3, pp. 546-552, 1983.

[12] D. V. Finch, "Cone beam reconstruction with sources on a curve," SIAM J. Appl. Math., vol. 45, no. 4, pp. 665-673, 1985.

[13] F. Noo, A. Wunderlich, G. Lauritsch, and H. Kudo, "On the problem of axial data truncation in the reverse helix geometry," in Proc. 10th Int. Meeting on Fully 3-D Image Reconstruc. Radiol. Nucl. Med., Beijing, China, Sep. 2009, pp. 90-93.

[14] S. Cho, D. Xia, C. A. Pellizzari, and X. Pan, "A BPF-FBP tandem algorithm for image reconstruction in reverse helical cone-beam CT," Med. Phys., vol. 37, no. 1, pp. 32-39, Jan. 2010.

[15] Z. Yu, F. Noo, F. Dennerlein, G. Lauritsch, and J. Hornegger, "FDKtype reconstruction algorithms for the reverse helical trajectory," in Proc. Nucl. Sci. Symp. Med. Imag. Conf. Rec., Valencia, Spain, Oct. 2011, pp. 3980-3985.

[16] Z. Yu, M. Andreas, M. Schönborn, F. Vogt, C. Köhler, G. Lauritsch, J. Hornegger, and F. Noo, "First experimental results on long-object imaging using a reverse helical trajectory with a C-arm system," in Proc. 2nd Int. Conf. Image Form. X-ray Comput. Tomogr., Salt Lake City, UT, Jun. 2012, pp. 364-367. 
[17] A. Rougee, C. L. Picard, Y. L. Trousset, and C. Ponchut, "Geometrical calibration for 3-D X-ray imaging," in Proc. SPIE, Med. Imag., Image Capture, Format., Display, Newport Beach, CA, Feb. 1993, vol. 1897, pp. 161-169.

[18] R. Fahrig and D. W. Holdsworth, "Three-dimensional computed tomographic reconstruction using a C-arm mounted XRII: Image-based correction of gantry motion nonidealities," Med. Phys., vol. 27, pp. 30-38, Jan. 2000.

[19] M. D. Silver, A. Sen, and S. Oishi, "Determination and correction of the wobble of a C-arm gantry," in Proc. SPIE, Med. Imag., Image Process., San Diego, CA, Feb. 2000, vol. 3979, pp. 1459-1468.

[20] K. Wiesent, K. Barth, N. Navab, P. Durlak, T. Brunner, O. Schuetz, and W. Seissler, "Enhanced 3-D-reconstruction algorithm for C-arm systems suitable for interventional procedures," IEEE Trans. Med. Imag., vol. 19, no. 5, pp. 391-403, May 2000.

[21] A. Maier, J.-H. Choi, A. Keil, C. Niebler, M. Sarmiento, A. Fieselmann, G. Gold, S. Delp, and R. Fahrig, "Analysis of vertical and horizontal circular C-arm trajectories," in Proc. SPIE, Med. Imag., Phys. Med. Imag., Lake Buena Vista, CA, Feb. 2011, pp. 79612 31-79 61238.

[22] N. Strobel, B. Heigl, T. Brunner, O. Schuetz, M. Mitschke, K. Wiesent, and T. Mertelmeier, "Improving 3-D image quality of X-ray C-arm imaging systems by using properly designed pose determination systems for calibrating the projection geometry," in Proc. SPIE, Med. Imag., Phys. Med. Imag., San Diego, CA, Feb. 2003, vol. 5030, pp. 943-954.

[23] F. Vogt, Geometry calibration for arbitrary trajectories Jul. 2008, Disclosure Number: IPCOM000172082D [Online]. Available: http://ip. com/IPCOM/000172082

[24] C. Mennessier, R. Clackdoyle, and F. Noo, "Direct determination of geometric alignment parameters for cone-beam scanners," Phys. Med. Biol., vol. 54, no. 6, pp. 1633-1660, Feb. 2009.

[25] M. Zellerhoff, B. Scholz, E.-P. Ruehrnschopf, and T. Brunner, "Low contrast 3-D reconstruction from C-arm data," in Proc. SPIE, Med. Imag.: Phys. Med. Imag., San Diego, CA, Feb. 2005, vol. 5745, pp. 646-655.
[26] M. John, R. Liao, Y. Zheng, A. Nöttling, J. Boese, U. Kirschstein, J. Kempfert, and T. Walther, "System to guide transcatheter aortic valve implantations based on interventional C-arm CT imaging," in Medical Image Computing and Computer-Assisted Intervention-MICCAI 2010. New York: Springer, 2010, pp. 375-382.

[27] S. Hoppe, F. Noo, F. Dennerlein, G. Lauritsch, and J. Hornegger, "Geometric calibration of the circle-plus-arc trajectory," Phys. Med. Biol., vol. 52, no. 23 , pp. 6943-6960, Nov. 2007.

[28] M. Mitschke and N. Navab, "Recovering the X-ray projection geometry for three-dimensional tomographic reconstruction with additional sensors: Attached camera versus external navigation system," Med. Image Anal., vol. 7, no. 1, pp. 65-78, 2003.

[29] O. Faugeras, Three Dimensional Computer Vision: A Geometric Viewpoint, 2nd ed. Cambridge, MA: MIT Press, 1993

[30] L. A. Feldkamp, L. C. Davis, and J. W. Kress, "Practical cone-beam algorithm," J. Opt. Soc. Am. A, vol. 1, no. 6, pp. 612-619, Jun. 1984

[31] G. Wang, T.-H. Lin, P.-C. Cheng, and D. M. Shinozaki, "A general cone-beam reconstruction algorithm," IEEE Trans. Med. Imag., vol. 12, no. 3, pp. 486-496, Sep. 1993.

[32] Date sheet of QRM-cone-beam-phantom [Online]. Available: http://www.qrm.de/content-/pdf/QRM-ConeBeam-Phantom.pdf QRM GmbH, Darfstrasse 4, 91096. Moehrendorf, Germany

[33] M. Remy-Jardin, J. Remy, F. Giraud, and C. Marquette, "Pulmonary nodules: Detection with thick-section spiral CT versus conventional CT," Radiol., vol. 187, no. 2, pp. 513-520, 1993.

[34] Z. Yu, A. Wunderlich, F. Dennerlein, G. Lauritsch, and F. Noo, "Line plus arc source trajectories and their R-line coverage for long-object cone-beam imaging with a C-arm system," Phys. Med. Biol., vol. 56, no. 12, pp. 3447-3471, May 2011.

[35] Z. Yu, F. Noo, G. Lauritsch, F. Dennerlein, and J. Hornegger, "Ellipse-line-ellipse source trajectory and its R-line coverage for long-object cone-beam imaging with a C-arm system," in Proc. SPIE, Med. Imag.: Phys. Med. Imag., San Diego, CA, Feb. 2012, vol. 8313, pp. 83 133E1-83 133E6. 